\title{
Jürgen Seifert Totalrevision: Drohung mit dem Verfassungsbruch
}

Noch in der Endphase der Auseinandersetzung um die Notstandsgesetzgebung, am 3. April 1968, äuferte der CDU-Abgeordnete Hans Dichgans in der Haushaitsdebatte des Bundestages: $*$ Wir haben uns niemals die Frage vorgelegt, ob das Grundgesetz als Ganzes den heutigen Bedürfnissen noch entspricht... ich möchte Ihnen vorschlagen, da $B$ wir einmal darüber nachdenken, ob es nicht richtig ist, etwa heute schon zu beschließen, im Jahre 1974 eine neue verfassunggebende Nationalversammlung einzuberufen, die von Grund auf noch einmal alle Fragen stellt, die gestellt werden müßten. « ${ }^{1}$

Dichgans ist kein Außenseiter. Im Januar 1969 stand er als Kandidat für den Bundestagspräsidenten zur Diskussion. Er kommt aus dem Management der Stahlindustrie und ist geschäftsführendes Vorstandsmitglied der Wirtschaftsvereinigung Eisen- und Stahlindustrie, Düsseldorf. Bekannt geworden ist er durch seine Intitiative, die akademische Freiheit durch Begrenzung der Studienzeit zu beschneiden.

Ralf Dahrendorf hat den Gedanken einer Verfassungsrevision durch eine Verfassunggebende Nationalversammlung in einer Zeitungskolumne als "sinnvoll « bezeichnet ${ }^{2}$. Theodor Eschenburg hat Dichgans nachdrüdklich unterstützz ${ }^{3}$, zunächst allerdings ohne Resonanz zu finden, da das Grundgesetz in Art. 79 die Vorausserzungen, unter denen Verfassungsänderungen zulässig sind, unmißverständlich umreißt. Eschenburg hat auch die Große Anfrage, die am 27. Juni 196842 Bundestagsabgeordnete der Union an die Bundesregierung richtete ${ }^{4}$, in diesen $\mathrm{Zu}-$ sammenhang gestelltr. Die Abgeordneten wollten von der Bundesregierung wissen, ob das föderative System noch »den Anforderungen genügt, die in unserer Zeit an einen demokratischen und sozialen Rechtsstaat in einem sich zusammenschließenden Kontinent gestellt werden müssen? « Die Anfrage stellt nicht nur die im Grundgesetz verankerte Kompetenzverteilung zwischen Bund und Ländern auf dem Gebiet der Gesetzgebung und der Verwaltung grundsätzlich in Frage, sondern auch die Zuständigkeiten auf dem Gebiet der Rechtssprechung und auf dem Gebiet des Gemeinderechts. Trotz der Frageform, die es den anfragenden Abgeordneten ermöglicht, sich nicht eindeutig festzulegen und ihre politi-

1 Bundestag, StenBer, 5. Wahlperiode, I66, Sitzung, S. 8723 (C).

- Ralf Dahrendorf, > Ober die Schwierigkeit, etwas in Bewegung zu setzen \&, in: Der Volkswirt, Jg. 22, Nr. 25 (21. Juni 1968), S. 16.

- Theodor Eschenburg, $\rightarrow$ Revision des Grundgeserzes? Anmerkungen zu einem bemerkenswerren Vorschlag*, in: Die Zeit, 28. Juni 1968, Nr. 26, S. 5; Eschenburg verweist dabei u. a. auf die gegenwärtigen Bestrebungen in der Schweiz; vgl. dazu Max Imboden, Verfassungsrevision als Weg in die Zukunft, Bern, г 966.

- Bundestagsdrucksache V/3099 (neu) vom 27. Juni 1968 , sbetr. Weiterentwidklung des föderativen Systems*; Antwort: Bundestagsdrucksache V/4002 vom 20. März 1969.

5 Theodor Eschenburg, s Noch einmal: Revision des Grundgesetzes. Zu einem Antrag von 45 CDU-Abgeordneten *, in: Die Zeit, 5. Juli 1968, Nr. 27, S. 7. 
schen Auffassungen nur anzudeuten, ist dieses von der Bundesregierung erst im März 1969 beantwortete Dokument ein interessanter Beleg für das gebrochene Verhältnis dieser Volksvertreter zu den Prinzipien des Grundgesetzes von 1949.

Im Dezember 1968 legte Hans Dichgans, von Hause aus Jurist, eine Konzeption vor, die einen Weg weist, die verfassungsrechtlichen Hindernisse (Art. 79 GG), die seinem Plan im Wege stehen und die auch Theodor Eschenburg bedauert hatte, auf seine Weise zu umgehen ${ }^{7}$. Er vertrat die Ansicht, das deutsche Volk im Sinne des Arr. 146 GG sei wheute das Volk der Bundesrepublik «. Er folgert daraus: $\gg$ Bundestag und Bundesrat können heute schon durch ein Gesetz nach Art. 79 eine neue verfassunggebende Nationalversammlung einberufen, die eine neue Verfassung gemäß Art. 146 ausarbeitet. «

Theodor Eschenburg, der in der Regel betont, Politikwissenschaftler und nicht Verfassungsjurist zu sein, will in dieser Frage die Auseinandersetzung vor dem Bundesverfassungsgericht $»$ nicht scheuen $\star^{8}$ und hat die von Dichgans vorgeschlagenen Umkehrung des ausschließlich für den Fall der Wiedervereinigung vorgesehenen Arr. ${ }_{4} 6$ ausdrücklich gutgeheißen. Eschenburg hat auch die Motive für seine Überlegungen verraten: Eine verfassunggebende Nationalversammlung würde »die Ausschaltung des Bundesrates von dieser Aufgabe bedingen $\star^{9}$; eine Grundgesetzänderung auf dem in der Verfassung gewiesenem Wege »wäre ein aussichtsloses Unternehmen. Eine $Z$ weidrittelmehrheit ist so schwer zu erreichen, daß die Gesamtrevision ein Fragment bleiben würde « ${ }^{10}$.

Weder Dichgans noch Eschenburg noch der Hamburger Offentlichrechtler Werner Thieme (der sich inzwischen der Kampagne für eine Totalrevision des Grundgesetzes angeschlossen hat ${ }^{11}$ ) hat sich die Mühe gemacht, konkret nachzuweisen, warum das Grundgesetz überholt sei. Deutlich wurde alierdings, daß bei einer Totalrevision nicht an eine Verstärkung demokratisch-plebiszitärer Elemente gedacht ist, die Helmut Lindemann gefordert hat ${ }^{12}$, sondern um die Durchsetzung zentralstaatlicher Strukturen. So meinte Eschenburg, der Föderalismus sei »gemessen an unserer gesellschaftlichen Entwidklung, anachronistisch «13. Er will unter anderem die Kulturhoheit der Länder beseitigen (faktisch heißt das: Aufhebung der verfassungsrechtlichen Sicherungen gegen ein Regierungsfernsehen); er erwägt, die Zuständigkeiten des Bundesverfassungsgerichts einzuschränken und ist für Bestimmungen, die sicherstellen, daß die »massiven Störungen, die wir an den Universitäten erleben «, nicht vom Demonstrationsrecht erfaßt werden ${ }^{14}$. Auch Thieme ist der Ansicht, der Föderalismus habe seine Existenzberechtigung verloren ${ }^{15}$. Noch deutlicher wird Thieme, wenn er sagt: $\gg$ Weder die Freiheit der Verbände, noch die der öffentlichen Meinung, noch die der Wissenschaft ist hinreichend begrenzt, gegen Mißbrauch gesichert.... Es geht ja auch nicht nur um die Gewährleistung der Freiheir, sondern ebenso um deren Grenzen im Interesse

\footnotetext{
8 a. a. O. (Anm. 3).

7 Hans Didhgans, $\gg$ Eine verfassunggebende Nationalversammlung? « in: Zeitschrift für Recritspolitik, Jg. 1, Heft 3 (Dezember 1968), S. 61-63.

$8 \rightarrow$ Für die Demokratie eine Diktatur auf Zeit? Spiegel-Gespräch mit Theodor Eschenburg*, in: Der Spiegel, Jg. 23, Nr. 8 (17. Februar 1969), S. 36-50, insb. S. 42.

a. 2. O. (Anm. S).

10 2. a. O. (Anm. 3.)

11 Werner Thieme, Für eine Totalrevision des Grundgesetzese, in: Zeitshrift für Rechtspolitik, Jg. 2, Heft 2 (Februar 1969), S. 32-33.

12 Helmut Lindemann, Das antiquierte Grundgesetz, Hamburg, 1966.

13 2. 2. O. (Anm. 8), S. $4^{8}$.

14 Ebd, S. so.

15 a. a. O. (Anm. I1), S. 33.
} 
der Allgemeinheit, im Interesse der Handlungsfähigkeit des Staates als Diener der Gesellschaft. *

Inhaltlich geht es also unter dem Titel Totalrevision des Grundgesetzes durch eine verfassunggebende Nationalversammlung um eine Beschneidung sogenannter $\gg$ Rechte gegen den Staat» und um einen Abbau des Föderalismus, d. h. um die Ubertragung wesentlicher Befugnisse der Länder (z. B. Polizeigewalt) auf den Bund; es geht um Zentralisation und um den Abbau regionaler Selbstverwaltung. Begrenzung der $*$ Freiheit der Verbändek heißt konkret: Einschränkung der Macht der Gewerkschaften.

Die Verfechter einer Totalrevision des Grundgesetzes stehen vorerst allein. Eine Tagung, zu der die Friedrich-Naumann-Stiftung Ende März 1969 nach BadenBaden eingeladen hatte ${ }^{18}$, zeigte, daß nicht nur die Länder argwöhnisch einem solchen Verfahren gegenüberstehen. Auch in der SPD scheint man nicht geneigt zu sein, durch die Rede von einer Totalrevision die Norstandsopposition neu zu beleben. In der Politisch-Sozialen Korrespondenz hat sich im Mai 1969 der Bonner Staatsrechtler Ulrich Scheuner, der in wesentlichen Verfassungsfragen häufig die Position der Bundesregierung vertreten hat, gegen eine Totalrevision gewandt ${ }^{17}$. Die Frankfurter Allgemeine Zeitung, die über Dichgans schon anläßlich seiner damals diskutierten Kandidatur zum Bundestagspräsidenten berichtet hatte, er habe w viele Fraktionsmitglieder mit radikalen Vorschlägen zur Verfassungsreform verstimmt $\alpha^{18}$ - hat an hervorgehobener Stelle auf Scheuners Artikel hingewiesen und dadurch ihre ablehnende Halrung zum Ausdruds gebracht ${ }^{19}$. In einer vom $Z$ weiten Deurschen Fernsehen am is. Mai 1969 gesendeten Disputation zwischen Hans Dichgans und Ulrich Scheuner hat Dichgans zwar die Forderung nach einer Totalrevision und den Weg über Art. 146 GG nicht aufgegeben, in der Sache jedoch merklich Zurückhaltung gezeigt. Dichgans wandte sich gegen eine Veränderung der Geserzgebungskomperenz des Bundes und plädierte letztlich nur für die Konstiruierung eines Länderrates, der neben den Bundesrat treten soll, und für eine Verbesserung des Gesetzgebungsverfahrens. Er erklärte: $\gg$ Alles, was ich als Reform unterbringen möchte, stößt sich an Art. 79 Abs. 3 nicht ${ }^{20} . *$

Noch deutlicher als Ulrich Scheuner betonte Bundesinnenminister Ernst Benda in einem Gedenkartikel zum 23. Mai 1969: „Für eine Verfassungsneuschöpfung - aber auch für eine Totalrevision der Verfassung - scheint mir ein wirklicher Anlaß nicht gegeben.* Bendas Begründung ist bestimmt durch die Erfahrungen des Kampfes um die Norstandsgesetze: „Ein solches Vorgehen würde vielmehr unnötig die Gefahr heraufbeschwören, die weitaus überwiegend bewährten Elemente unserer geltenden Verfassungsordnung aufs Spiel zu setzen und den ihr zugrunde liegenden demokratischen Konsensus zu verunsichern.* Benda sprach

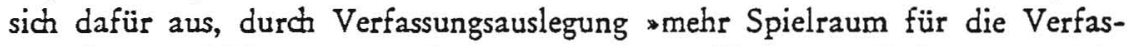
sungsfortentwidklung $\mathrm{zu}$ gewinnen «; wenn von Verfassungsänderungen nicht abgesehen werden könne, so sollten $»$ die jeweils anstehenden Verfassungsände-

to S. dazu Roderich Reifenrath, Alle Rufe nach einer Totalrevision sind unsinniga, in: Frankfurter Rundschau, 3I. März I969, Nr. 76, S. 3.

17 Ulrich Scheuner, $\rightarrow$ Revision des Grundgesezzes? in: Politisch-Soziale Korrespondenz, Jg. 18 , Heft $g$ (s. Mai 1969), S. 2-3.

$18 \rightarrow$ Die CDU unter Zeirdrudk: Wer wird Gerstenmeiers Nachfolger?. in: Frankfurter Allgemeine Zeitung, 27. Januar 1969, Nr. 22, S-Ausgabe S. r.

19 -Gegen Enneuerung des Grundgeserzes*, in: Frankfurter Allgemeine Zeitung, 30. April 1969, Nr. 100, S-Ausgabe S. I.

so Zweites Deutsches Fernsehen, slst das Grundgesetz tabu?* (Diskussion zwischen Hans Dichgans und Ulrich Scheuner in der Sendereihe sZur Saches), is. Mai 1969. 
rungen« koordiniert und »nach Möglichkeit an einer Gesamtkonzeption« orientiert $\operatorname{sein}^{202}$.

Die Auseinandersetzung ist nicht einfach als akademische Kontroverse abzutun. Denn der Vorstoß geht von einem Bundestagsabgeordneten aus und der Bundestag ist diesem Plan, Art. 79 GG einfach zu unterlaufen, bis heute nicht entgegengetreten. $Z$ u denken sollte auch geben, daß vor allem Zeitungen und Zeirschriften, die als liberal angesehen werden, ihre Spalten der Kampagne für eine Totalrevision zur Verfügung stellten, ohne daß die Gegenposition in gleicher Weise zu Wort kam. Ist es nicht ein Symptom, daß die veranstaltete öffentliche Meinung, die in der Regel Räre schlechthin als verfassungsfeindlich einzuordnen sucht, die Aufforderung unbeachtet hingenommen hat, die Bestandsgarantie zu liquidieren, die den Kern des Grundgesetzes schützt?

Die Umgehung der Schranken des Arr. 79 Abs. 3 GG durch den ausschließlich für eine Wiedervereinigung vorgesehenen Art. I46 GG überschreitet nicht nur - wie es Scheuner zurückhaltend nennt - »die Grenzen sinngebender Verfassungsinterpretation ${ }^{21}$, sondern demontiert die Bestandssicherung, die von den Schöpfern des Grundgesetzes als Garantie eingebaut wurde, um eine Transformation der Verfassungsordnung des Grundgesetzes in ein autoritäres oder totalitäres System zu verhindern. Der Vorstoß des Abgeordneten Dichgans richtet sich gegen die Verfassungsbestimmung, die ausschließen soll, daß erneut ein offenes faschistisches System unter dem Mantel der Scheinlegalität errichtet werden kann. Der CDU-Abgeordnete Hans Dichgans kann somit beanspruchen, als erster allen Gegnern des Grundgeserzes den Weg gewiesen zu haben, wie die Grundprinzipien des demokratischen und sozialen Rechtsstaats auf scheinlegalem Wege zu beseitigen sind.

Bedingt wird eine derartige Verhaltensweise nicht zuletzt durch alle diejenigen Verfassungstheorien, die eine Verfassung nicht anders behandeln als eine StraBenverkehrsordnung, die laufend den Erfordernissen des Verkehrs anzupassen ist, und die es versäumen, Verfassungen und Verfassungsbestimmungen als das Produkt divergierender und konkurrierender politischer und gesellschaftlicher Interessen zu interpretieren.

Moderne Verfassungen entstehen - wie es der Engländer Kenneth Wheare einmal treffend bezeichnete - unter Umständen, in denen $\star_{a}$ break with the past and the need for a fresh start * geboten ist ${ }^{22}$. Sie sind immer Abgrenzung, Frontstellung und Bastion gegen etwas, das nicht - oder nicht mehr - sein soll. So entstand das Grundgesetz aus der Gegenposition gegen den autoritären und faschistischen Staat und gegen die Herrschaftssysteme kommunistischer Volksrepubliken. Diese Gegenposition fand ihren Niederschlag in Art. 79 Abs. 3, durch den unter anderem die Würde des Menschen, die Grundsätze der Demokratie, der Sozialstaatlichkeit, der Rechtsstaatlichkeit und der Verteilung der Macht zwischen Bund und Länder zu unantastbaren Verfassungsgrundsätzen erklärt worden sind. Für den Parlamentarischen Rat, der sich sehr wohl bewußt war, daß das deursche Volk den Faschismus nicit von Innen heraus überwunden hatte, war es - auch auf Grund der Erfahrungen am Ende der Weimarer Republik - eine Selbstver-

200 Ernst Benda, Fundament der Freiheirlichkeite, in: Deutschland-Union-Dienst. Das Widtigste der Woche (Pressedienst der Christich Demokracischen und Christich-Sozialen Union), 23. Mai r 969, Nr. 20, S. 3; auch auf diese Außerung wies die FAZ an hervorgehobener Stelle hin: - Benda lehnt cocale Revision des Grundgesetzes abe, in: Frankfurter Allgemeine Zeitung, 21. Mai 1969, Nr. 116, S-Ausgabe, S. r.

:1 a. 2. O. (Anm. 17), S. 3 .

22 K. C. Wheare, Modern Constitutions, (Oxford Papercadss University Series, Bd. II) London, New York, Toronto, 1966, S. 6. 
ständlichkeit, den Kern der Verfassung besonders zu sichern. Art. 79 GG erleichtert es, den Verfassungsbruch als Verfassungsbruch zu entlarven.

Dichgans zeigt, daß für ihn der Kern des Grundgesetzes nicht mehr wa break with the past $\alpha$ und nicht mehr durch die skizzierte Frontstellung geprägt ist. Sonst wäre es ihm unmöglich zu fragen, »was eigentlich die Generation von 1949 berechrigen konnte, ihre Enkel und Urenkel, bis in das 3. Jahrtausend der Zeitrechnung hinein, an dieses System zu binden, auch wenn sie mit überwältigender Mehrheit ein anderes für besser halten ${ }^{23}$ ? Die konkrete Fronstellung, die den Abgeordneten Dichgans bestimmt, zeigt sich beispielsweise in dem unter seiner Initiative vorgelegten Gesetzentwurf, durch den unter anderem die von Gerichten verhängten Ordnungsstrafen von drei Tagen auf sechs Wochen und von ro00,- DM auf ro o00,-DM heraufgesetzt werden sollen²4.

Die Versuche, das Erfordernis der Zustimmung einer $Z_{\text {weidrittelmehrheit von }}$ Bundestag und Bundesrat zu einer konkreten Verfassungsänderung zu umgehen, verkennt, daß dieses Erfordernis zu den Voraussetzungen gehört, unter denen die rivalisierenden politischen und gesellschaftlichen Kräfte die Verfassung anerkennen. Für diese Kräfte ist die Verfassung kein bloßes Vereinsstatut, sondern ein Waffenstillstand, ein Kompromiß (der den Rahmen setzt, innerhalb dessen in Zukunft die politischen Auseinandersetzungen ausgetragen werden sollen) oder eine Koalitionsvereinbarung über ein Condominium. Freilich klammert die deutsche Verfassungslehre diese realen Voraussetzungen einer Verfassung in der Regel aus und verdeckt sie durch pathetische Formeln, die beispielsweise Verfassunggebung als Zukuntsplanung definieren oder das Wesen einer Verfassung bestimmen als die existentielle Entscheidung eines Volkes über die Art und Weise des eigenen Seins. So ist es auch kein Zufall, daß eine umfassende Monographie über die Bedeutung qualifizierter Mehrheiten bei Verfassungsänderungen und die Zurückführung der unterschiedlichen Formen dieser Bestandsgarantien auf die beteiligten politisch-gesellschaftlichen Kräfte nicht vorliegt.

Der Vorstoß von Dichgans und Eschenburg (zusammen mit der Großen Anfrage von 42 Abgeordneten der Union) läßt erkennen, in welchem Umfang politische Führungsgruppen der Bundesrepublik wesentliche Verfassungsbestimmungen des Grundgeserzes - trotz Notstandsverfassung - noch immer als ein Hemmnis ansehen, ihre Politik zu verwirklichen. Die Rede von einer verfassunggebenden Nationalversammlung, die Abwertung von Verfassungskompromissen als Flikkenteppich und die Forderung nach einer Verfassung aus einem Guß mache deurlich, in welchem Umfang eine Liquidation des Grundgesetzes und des politischen Denkens der "Großväter von I947 " (so polemisiert Hans Dichgans ${ }^{25}$ ) angestrebt wird. Dennoch genügt es nicht, sich demgegenüber auf das gute alte Recht, auf den Geist der Nachkriegszeit oder dergleichen zu berufen.

Der Angriff auf die bundesstaarliche Struktur und auf die Bestandsgarantie des Art. 79 Abs. 3 GG ist nicht primär ökonomisch bedingt. So denkt beispielsweise in dem ganz anderen ökonomischen $Z$ wängen unterworfenen System der Vereinigten Staaten niemand daran, den Föderalismus zu beseitigen. $Z \mathbf{u}$ denken geben sollte auch die Tatsache, daß der gegenwärtige Angriff sowohl gegen die Formen als auch die Grenzen verfassungsgemäßer Verfassungsänderungen gerade nicht von den betont konservativen und reches stehenden politischen

39. a. O. (Anm. 7), S. 62 .

94 Bundestagsdrucksache V/3743 (Entwurf eines Gesetzes zur Anderung des Gerichtsverfassungsgesetzes und des Strafgesezzbuches); vgl. dazu die Ausführung von Hans Didgans Bundestag, StenBer, 5. Wahlperiode, 2 Ir. Sitzung, 24. Januar I969, S. I I442 (B) - I I443 (D).

as Hans Didhgans, Das Unbehagen in der Bundesrepublik. Ist die Demokratie am Ende? Düsseidorf, Wien, 1968, S. 275; ähnlich in der Fernsehdiskussion am 15. Mai 1969, a. a. O. (Anm. 20). 
Kräften getragen wird. Diese reden weniger, sie versuchen dagegen ihre tatsächliche Machıstellung in den realen politisch-gesellschaftlichen Verfassungspositionen zu verbessern. - Unzureichend ist auch die Erklärung von Friedrich Karl Fromme, der die Vorstöße des Abgeordneten Dichgans mit dem Sazz abwertet, sie »mögen ein wenig modischer Attitüde oder auch der von begierigen Massenmedien genährten Lust am ,Vorschlage entspringen ${ }^{253}$. Die Forderungen nach einer Totalrevision des Grundgesetzes haben - abgesehen von dem vorherrschenden formalen Verfassungsdenken, das Verfassungsbestimmungen loslöst vom politisch-gesellschaftlichen Kräftefeld, - im wesentlichen politische Gründe. Die bundesstaatliche Struktur ist für die CDU/CSU ein Hemmnis, insbesondere seitdem die Sozialdemokraten in den Ländern Machtpositionen erlangten, die es der SPD ermöglichen, über den Bundesrat stärker als je zuvor auf die Bundespolitik Einfluß zu nehmen. Noch ist nicht abzusehen, welche Bedeutung diesen Positionen der SPD in den Ländern - und damit auch im Bundesrat - nach den Bundestagswahlen zukommen wird. Es liegt auf der Hand, daß von der CDU/CSU eine Aushöhlung der Machtpositionen der SPD in den Ländern öffentlich nicht unter Hinweis auf die bedrohte Stellung der Unionsparteien geführr werden kann. Die Auseinandersetzung wird daher mit der Argumentation geführr, daß *die Zeit» größere Effizienz und Schlagkraft (Hinweis auf Verbrechensbekämpfung), mehr Einheitlichkeit und Gleichklang (Hinweis auf die konträren Bildungspläne und auf das Fehlen eines gemeinsamen Vorgehens in Sachen Hochschulreform) und mehr Gemeinsamkert in einem sich zusammenschließenden Kontinent fordere. Es wird der Eindrudk erweckt, daß die Probleme nur dann gelöst werden können, wenn an die Stelle der Tätigkeir diverser Landesministerien eine Bundeszuständigkeit trete. Doch was inhaltlich anders werden soll, wird nicht gesagt. Man bewegt sich im Bereich der Zuständigkeiten. Eingriffe in die bundesstaatliche Struktur jedoch bedeuten stets mehr Zentralisation, größere Unabhängigkeit der Unionsparteien von den sozialdemokratischen Gegenpositionen in den Ländern und damit zugleich verstärkten Einfluß der Bürokratien des Bundes. In diesem Zusammenhang wird deutlich, $\mathrm{da} ß$ auch der gegenwärtige Angriff auf den Kern der 1949 geschaffenen und für unantastbar erklärten Verfassungsgrundsäzze Ausdruck der Tendenz ist, die nach der Verfassungsurkunde von 1949 rechtsstaatliche und auf dem Grundsatz der Machtverteilung aufbauende Verfassungsordnung der Bundesrepublik zu transformieren in das Verfassungssystem eines autoritären Systems.

Wenn sich Hans Dichgans und Theodor Eschenburg mit ihrer Konzeption durchsetzen sollten, dann würde das bewreisen, daß wir von einem Kartell von Politikern beherrscht werden, indem die Konkurrenz aufgehoben ist und das im Bundestag und im Bundesrat eine Zweidrittelmehrheit kontrolliert. Davon aber gehen auch Dichgans und Eschenburg nicht aus, wie ihr krampthaftes Bestreben zeigt, den Bundesrat aus der Endentscheidung über die Totalrevision auszuschalten.

Der Vorstoß von Dichgans und Eschenburg erfüllt deshalb - unabhängig von den Motiven der Agierenden - eine andere Funktion: Je lautstärker eine Totalrevision des Grundgesetzes gefordert wird, desto größer wird die Chance, Teilreformen durchzusetzen. Aufschlußreich ist in diesem Zusammenhang die Stellungnahme von Ulrich Scheuner ${ }^{28}$. Scheuner, nur bedingt Gegner einer Totalrevi-

ssa Friedrich Karl Fromme, \as Grundgesetz und die Lehren von Weimars, in: F. A. Krummacher, so Jahre deutsdie Republik, Frankfurt am Main, 1969, zitiert nach dem Vorabdruds: -Erst Zwanzigjührig und schon veraltet? in: Frankfurter Allgemeine Zeitung, 20. Mai 5969, Nr. IIs, S-Ausgabe, S. 19.

28 a. 2. O. (Anm. 17), S. 3. 
sion, unterstützt die Befürworter einer Totalrevision in ihrem Ziel. Er meint, "die schrittweise Anpassung « sei »der richtige Weg*, weil die Zeit nicht »schon [!!!] für eine grundsätzliche Neuordnung reif $\star$ und »der gegenwärtige Zeitpunkt* nicht für eine „Neuformung « geeignet sei. Scheuner empfiehlt zu warten, bis $*$ das zuweilen allzu starke auf die Verteidigung des Besitzstandes gerichtete Verhalten der Länder mehr und mehr den Rüdkhalt der öffentlichen Meinung verliert « ${ }^{27}$. Auch Bundesinnenminister Ernst Beada erklärte in seiner Antwort auf die Große Anfrage zum Föderalismus, Verfassungsänderungen seien sin Zukunft auch im föderativen Bereich unvermeidlich $\alpha$, wandte sich gegen das Beharren *auf überholten Besitzständen und versuchte, die von ihm entwidkelten Vorstellungen der Zusammenarbeit von Bund und Ländern als »kooperativen Föderalismus und »kooperativen Bundesstaat « schmadkhaft zu machen². Die Drohung, die der von Dichgans dargelegten Interpretarion von Art. I46 GG zu Grunde liegt, durch Anwendung dieser Bestimmung die Länder zumindest in der Endentscheidung ausschalten zu können, ist ein Mittel, widerborstige Gegenkräfte dazu zu bringen, Zugeständnisse zu machen. So stimmte die SPD der Notstandsverfassung unter anderem mit der Begründung zu, nur durch eine Notstandsverfassung könnte die von der CDU/CSU angedrohte Anwendung eines sogenannten übergesetzlichen Notstandsrechts oder eine Übertragung alliierter Notstandsbefugnisse auf die Bundesregierung verhindert werden. Die SPD geriet in diese Situation, weil sie dem auf diese Weise angedrohten Verfassungsbruch nicht entschieden entgegen trat. Ob die Drohung mit einer verfassunggebenden Nationalversammlung (in der der Bundesrat ausgeschaltet ist) die einer Totalrevision widerstrebenden Kräfte dazu bringen wird, partiellen Verfassungsänderungen zuzustimmen, wird deshalb nicht zuletzt davon abhängen, ob das Konzept des Abgeordneten Dichgans als das entlarvt wird, was es ist: als Auforderung zum Verfassungsbruch.

7 Am I5. Mai 1969 erklärte er (a. a. O., Anm. 20): DEs wird sicherlich einmal der Zeitpunkt kommen, an dem man eine Generalbereinigung wird durchführen müssen.e

29 Bundestagsdrucksache V/4002 vom 20. März 1969, Betr.: Weiterentwidklung des föderativen Systems. 